\title{
Regional Anesthesia
}

Editors

NABIL M. ELKASSABANY

EDWARD R. MARIANO

\section{ANESTHESIOLOGY CLINICS}

www.anesthesiology.theclinics.com

Consulting Editor

LEE A. FLEISHER

September 2018 • Volume 36 • Number 3 